\title{
Positionspapier zur Lipidtherapie bei Patienten mit Diabetes mellitus
}

\author{
Eine gemeinsame Stellungnahme der Kommission Fettstoffwechsel sowie der AG Herz \\ und Diabetes der Deutschen Diabetes Gesellschaft (DDG), der Sektion Diabetologie und \\ Stoffwechsel der Deutschen Gesellschaft für Endokrinologie (DGE), der AG Herz und \\ Diabetes der Deutschen Gesellschaft für Kardiologie (DGK) und der gemeinsamen AG \\ Herz - Hormone - Diabetes der DGK, DGE und DDG
}

\author{
Autoren \\ Katharina S. Schütt ${ }^{5}$, Andreas Zirlik ${ }^{7}$, Dirk Müller-Wieland ${ }^{5}$ \\ Institute \\ 1 Medizinische Klinik IV - Großhadern, Klinikum der \\ Universität München, München \\ 2 Medizinische Klinik III, Universitätsklinikum Carl Gustav \\ Carus, Dresden \\ 3 Deutsches Zentrum für Diabetesforschung (DZD e.V.), \\ Neuherberg \\ 4 Poliklinik für Endokrinologie, Diabetologie und \\ Präventivmedizin, Uniklinik Köln, Köln \\ 5 Medizinische Klinik I - Kardiologie, Angiologie und \\ Internistische Intensivmedizin, Universitätsklinikum RWTH \\ Aachen, Aachen \\ 6 Endokrinologikum Hamburg, Hamburg \\ 7 Klinik für Kardiologie und Angiologie I, Universitäts- \\ Herzzentrum Freiburg
}

Klaus G. Parhofer ${ }^{1}$, Andreas L. Birkenfeld ${ }^{2,}{ }^{3}$, Wilhelm Krone ${ }^{4}$, Michael Lehrke ${ }^{5}$, Nikolaus Marx ${ }^{5}$, Martin Merkel ${ }^{6}$,

Bibliografie

DOI https://doi.org/10.1055/a-0598-7355

Diabetologie 2018; 13 (Suppl 2): S209-S213

(c) Georg Thieme Verlag KG, Stuttgart · New York

ISSN 1861-9002

Korrespondenzadresse

Prof. Dr. med. Klaus G. Parhofer

Klinikum der Universität München

Medizinische Klinik und Poliklinik IV - Großhadern,

Marchioninistraße 15, 81377 München

Klaus.Parhofer@med.uni-muenchen.de

\section{Präambel}

Patienten mit Diabetes mellitus haben in aller Regel ein deutlich erhöhtes kardiovaskuläres Risiko. Deswegen ist die Lipidtherapie bzw. eine Senkung des LDL-Cholesterins basierend auf einer Risikostratifizierung ein integraler Bestandteil der Diabetestherapie; daher ist folgende Positionierung auch als themenbezogene Ergänzung der jährlich aktualisierten Empfehlung zur Therapie des Typ-2-Diabetes zu sehen und soll ebenfalls in Zukunft jährlich zusammen mit den Praxisempfehlungen der DDG aktualisiert werden.

Die Positionierung basiert auf den kürzlich diesbezüglich erschienenen Leitlinien und Empfehlungen der Europäischen Gesellschaft für Kardiologie (ESC), der Europäischen Gesellschaft für Atherosklerose (EAS), der Amerikanischen Gesellschaft Klinischer Endokrinologen (AACE), der Amerikanischen Diabetesgesellschaft (ADA) und der amerikanischen Nationalen Lipid-Gesellschaft (NLA) [1 - 5].

Daher ist dieses Positionspapier als eine kurze, aktuelle, klinisch orientierte Handlungsempfehlung bei Patienten mit Diabetes zu verstehen; für vertiefende Erläuterungen zu Fettstoff- wechselstörungen bei Diabetes wird auf die angegebene Literatur verwiesen.

\section{Stratifizierung des kardiovaskulären Risikos}

Patienten mit Diabetes mellitus haben in aller Regel ein deutlich erhöhtes kardiovaskuläres Risiko [6]. Dennoch wird empfohlen, dieses auch weiter zu differenzieren. Dabei kommen dieselben Risikofaktoren wie bei Patienten ohne Diabetes zum Tragen ( $\triangleright$ Tab. 1). Zu beachten ist, dass sich das Vorliegen mehrerer Risikofaktoren überadditiv auf das Gesamtrisiko auswirkt [7]. Das geschätzte Gesamtrisiko ist eine wesentliche Determinante, ob und gegebenenfalls wie intensiv eine lipidsenkende Therapie durchgeführt werden soll.

\section{Lipiddiagnostik}

Als Basis werden die Bestimmung von Gesamtcholesterin, LDL-Cholesterin, HDL-Cholesterin und Triglyzeriden sowie die Berechnung des Non-HDL-Cholesterin-Spiegels empfohlen. Einmalig sollte die 
- Tab. 1 Weiter zu berücksichtigende Risikofaktoren.

\begin{tabular}{|l|l|l|l|l|l|l}
\hline Risikofaktor & Kommentar \\
\hline $\begin{array}{l}\text { Positive Familienanamnese für } \\
\text { Vorzeitige Atheroskleroseereignisse }\end{array}$ & $\begin{array}{l}\text { Eigentlich nur bei Atherosklerose vor dem 55. bzw. 65. Lebensjahr bei Männern bzw. Frauen; diese Altersgrenze } \\
\text { ist derzeit nicht evidenzbasiert und sollte in Zukunft angesichts der zunehmenden Lebenserwartung evtl. nach } \\
\text { unten verschoben werden. }\end{array}$ \\
\hline Nikotinabusus & Zahl der „Packyears“ ist relevant. \\
\hline Eingeschränkte Nierenfunktion & Die Einschränkung der Nierenfunktion führt dosisabhängig zu einer Steigerung des Atheroskleroserisikos. \\
\hline Hypertriglyzeridämie & $\begin{array}{l}\text { Unabhängiger Risikofaktor, wahrscheinlich auch als Indikator für erhöhtes Non-HDL-Cholesterin mit athero- } \\
\text { genen Remnant-Partikeln }\end{array}$ \\
\hline HDL-Cholesterin-Erniedrigung & Unklar, ob unabhängiger Risikofaktor oder Indikator (meist gleichzeitig bestehende Hypertriglyzeridämie) \\
\hline Hypertonus & $>140 / 90$ mmHg oder unter antihypertensiver Medikation \\
\hline
\end{tabular}

- Tab. 2 Klassifikation der Lipidstoffwechselstörungen.

\begin{tabular}{|l|l|l|l|l|l|}
\hline Lipidstoffwechselstörung & Cholesterin & Triglyzeride & LDL-Chol & HDL-Chol & Non-HDL-Chol \\
\hline LDL-Hypercholesterinämie & $\uparrow$ & $\mathrm{n}$ & $\uparrow$ & $\mathrm{n}$ & $\uparrow$ \\
\hline Hypertriglyzeridämie & $\uparrow$ & $\uparrow$ & $\mathrm{n}$ & $\downarrow$ & $\uparrow$ \\
\hline Kombinierte Hyperlipoproteinämie & $\uparrow$ & $\uparrow$ & $\uparrow$ & $\downarrow$ & $\uparrow$ \\
\hline Isolierte HDL-Cholesterin-Erniedrigung & $\mathrm{n}$ & $\mathrm{n}$ & $\downarrow$ \\
\hline Lipoprotein(a)-Erhöhung & Kann isoliert oder in Kombination mit jeder Fettstoffwechselstörung auftreten. \\
\hline
\end{tabular}

- Tab. 3 Behandlungsziele von Fettstoffwechselstörungen.

\begin{tabular}{|l|l|l|}
\hline Behandlung & Klinischer Effekt & Evidenz \\
\hline Absenkung LDL-Cholesterin & Reduktion Atheroskleroseereignisse & gesichert \\
\hline Absenkung Non-HDL-Cholesterin & Reduktion Atheroskleroseereignisse & gesichert \\
\hline Absenkung Lipoprotein(a) & Reduktion Atheroskleroseereignisse & vermutet \\
\hline Absenkung Triglyzeride & Reduktion Atheroskleroseereignisse & vermutet \\
\hline Absenkung stark erhöhter Triglyzeride & Reduktion Häufigkeit akuter Pankreatitiden & vermutet/gesichert \\
\hline
\end{tabular}

Bestimmung des Lipoprotein(a)-Werts erfolgen. Besteht keine Hypertriglyzeridämie und wird das LDL-Cholesterin direkt bestimmt, kann die Bestimmung im nicht nüchternen Zustand erfolgen [8]. Wird das LDL-Cholesterin durch die Friedewald-Formel rechnerisch ermittelt, sollte der Patient nüchtern sein, da der Triglyzeridspiegel in die Kalkulation mit eingeht. Eine genetische Diagnostik ist für die klinische Praxis bei hohem Verdacht auf Familiäre Hypercholesterinämie gerechtfertigt, wenn dies Folgen für die Indikation und Therapiestrategie hat.

\section{Lipid-Phänotyp}

Es wird zwischen Hypercholesterinämie, Hypertriglyzeridämie und kombinierter Hyperlipidämie unterschieden. Für die klinische Praxis müssen sekundäre Ursachen ausgeschlossen bzw. behandelt und es muss an wichtige primäre Störungen, z. B. Familiäre Hypercholesterinämie, gedacht werden ( $\bullet$ Tab. 2). 
- Tab. 4 Lipidzielwerte bei Patienten mit Diabetes mellitus.

\begin{tabular}{|c|c|c|c|c|}
\hline \multirow[b]{2}{*}{ Risikogruppe } & \multirow[b]{2}{*}{ Definition } & \multirow{2}{*}{$\begin{array}{l}\text { Primäres Ziel } \\
\text { LDL-Chol }\end{array}$} & \multicolumn{2}{|l|}{ Sekundäre Ziele } \\
\hline & & & Non-HDL-Chol & AроB \\
\hline Sehr hohes Risiko & $\begin{array}{l}\text { Nachgewiesene Atheroskleroseerkrankung und/ } \\
\text { oder zusätzliche schwerwiegende Risikofaktoren }{ }^{1} \\
\text { und/oder Endorganschaden }{ }^{2}\end{array}$ & $\begin{array}{l}<70 \mathrm{mg} / \mathrm{dl}(1,8 \mathrm{mmol} / \mathrm{l}) \text { und } \\
\geq 50 \% \text { Absenkung vom Ausgangswert }\end{array}$ & $\begin{array}{l}<100 \mathrm{mg} / \mathrm{dl} \\
(2,6 \mathrm{mmol} / \mathrm{l})\end{array}$ & $<80 \mathrm{mg} / \mathrm{dl}$ \\
\hline Hohes Risiko & $\begin{array}{l}\text { Ohne nachgewiesene Atheroskleroseerkrankung } \\
\text { und ohne zusätzliche Risikofaktoren }{ }^{1} \text { und ohne } \\
\text { Endorganschaden }{ }^{2}\end{array}$ & $\begin{array}{l}<100 \mathrm{mg} / \mathrm{dl}(2,6 \mathrm{mmol} / \mathrm{L}) \text { und } \\
\geq 50 \% \text { Absenkung vom Ausgangswert }\end{array}$ & $\begin{array}{l}<130 \mathrm{mg} / \mathrm{dl} \\
(3,4 \mathrm{mmol} / \mathrm{l})\end{array}$ & $<100 \mathrm{mg} / \mathrm{dl}$ \\
\hline
\end{tabular}

\section{Behandlung von Fettstoffwechselstörungen bei Patienten mit Diabetes mellitus}

Das im Vordergrund stehende Ziel der Behandlung ist die Reduktion des erhöhten kardiovaskulären Risikos von Patienten mit Diabetes mellitus. Wichtigste Maßnahme ist dabei die Absenkung des LDL-Cholesterins. Weiter kann durch die Absenkung exzessiv erhöhter Triglyzeridwerte das Risiko für akute Pankreatitiden reduziert werden. Eine Normalisierung erhöhter Triglyzeridwerte kann daneben die Blutzuckereinstellung verbessern ( $\triangleright$ Tab. 3).

\section{Therapiestrategien mit dem Ziel, LDL-Cholesterin-Werte abzusenken}

Entsprechend den Empfehlungen der europäischen Fachgesellschaften erfolgt die Senkung der LDL-Cholesterin-Spiegel „zielwertorientiert“ unter Berücksichtigung des kardiovaskulären Risikos [1]. Dabei werden lediglich 2 Kategorien unterschieden, die für Patienten mit Diabetes mellitus Typ 1 und Typ 2 gleichermaßen gelten

$(\triangleright$ Tab. 4):

- Patienten mit sehr hohem Risiko (Patienten mit Diabetes und nachgewiesener Atheroskleroseerkrankung und/oder zusätzlichen Risikofaktoren und/oder Endorganschaden)

- Patienten mit hohem Risiko (Patienten mit Diabetes ohne nachgewiesene Atheroskleroseerkrankung und ohne zusätzliche Risikofaktoren und ohne Endorganschaden)

Als sekundäre Zielwerte gelten die Konzentrationen von Non-HDLCholesterin und Apolipoprotein B. Dies spiegelt die Tatsache wider, dass vermutlich alle Apolipoprotein-B-haltigen Lipoproteine atherogen sind [9]. Der Non-HDL-Cholesterin-Wert (= Gesamtcholesterin minus HDL-Cholesterin) reflektiert dies ebenfalls näherungsweise und erfasst neben LDL-Cholesterin auch VLDLCholesterin und Remnant-Cholesterin. Der Non-HDL-CholesterinZielwert ist deshalb bei Patienten mit Hypertriglyzeridämie oder gemischter Hyperlipidämie (also typischerweise bei Patienten mit Diabetes mellitus) relevant. Bei Normotriglyzeridämie liegt die VLDL-/Remnant-Cholesterin-Konzentration bei $<30 \mathrm{mg} / \mathrm{dl}$ (entsprechend einem Triglyzeridwert von ca. $150 \mathrm{mg} / \mathrm{dl}$ ), weshalb
Definition des LDL-C-Ziels

(Abschätzung des kardiovaskulären Absolutrisikos)

LSM + potentes Statin

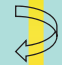

durch

Endpunkt-

studien

abgedeckt

Steigerung der Statin-Dosis

$\$$

Kombination mit Ezetimib

Ziel nicht erreicht? nächste Therapiestufe

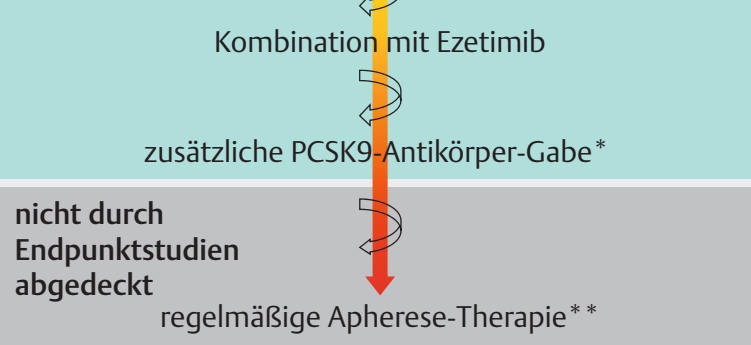

- Abb. 1 Therapiealgorithmus zur Erreichung der LDL-CholesterinZielwerte. LSM: Lebensstilmaßnahmen; * entsprechend den Vorgaben des G-BA; * * nach individueller Nutzen-Risiko-Abwägung.

Non-HDL-Cholesterin-Zielwerte jeweils um $30 \mathrm{mg} / \mathrm{dl}$ über dem LDL-Cholesterin-Zielwert liegen ( $\bullet$ Tab. 4). Bei Patienten, die zwar den LDL-Cholesterin-Zielwert, nicht aber den Non-HDL-Cholesterin-Zielwert erreichen, kann der Non-HDL-Cholesterin-Wert erreicht werden, indem entweder der Triglyzeridspiegel abgesenkt (Reduktion von VLDL-/Remnant-Cholesterin) oder das LDL-Cholesterin weiter reduziert wird.

Ergänzend soll erwähnt werden, dass die Amerikanische Diabetesgesellschaft (ADA) lediglich die Kriterien Alter (unter/ über 40 Jahre) und Vorliegen einer Atheroskleroseerkrankung (ja/ nein) berücksichtigt [5]. Dabei erhalten alle Patienten mit Atheroskleroseerkrankung eine hohe Dosis eines Statins (Atorvastatin $40-80 \mathrm{mg} / \mathrm{d}$ oder Rosuvastatin $20-40 \mathrm{mg} / \mathrm{d}$ ) und können zusätzlich mit Ezetimib und PCSK9-Inhibitoren behandelt werden, wenn der LDL-Cholesterin-Wert über $70 \mathrm{mg} / \mathrm{dl}$ bleibt. Von den Patienten ohne Atheroskleroseerkrankung erhalten die unter 40 Jahren nicht generell ein Statin und über 40-Jährige eine 
- Tab. 5 Therapiestrategien bei erhöhten Triglyzeriden.

\begin{tabular}{|c|c|}
\hline Maßnahme & Kommentar \\
\hline LDL-Cholesterin Zielwerterreichung & Immer; dazu ist normalerweise Statingabe notwendig. \\
\hline Non-HDL-Zielwerterreichung & $\begin{array}{l}\text { Wenn möglich; dazu entweder weitere LDL-Cholesterin-Absenkung oder Absenkung von VLDL-/ } \\
\text { Remnant-Cholesterin (und damit Triglyzeridabsenkung). }\end{array}$ \\
\hline Lebensstilmaßnahmen & Immer, da dadurch meist die Hypertriglyzeridämie deutlich verbessert werden kann. \\
\hline Blutzuckereinstellung & Immer, da dadurch meist die Hypertriglyzeridämie deutlich verbessert werden kann. \\
\hline Fibrate & $\begin{array}{l}\text { Einzelabwägung, evtl. nach Erreichung der LDL-Cholesterin-Zielwerte bei sehr hohem Risiko und weiter } \\
\text { bestehender Hypertriglyzeridämie;' zurückhaltender Einsatz, da bisher keine überzeugenden Endpunkt- } \\
\text { studien in Kombination mit Statinen; cave: erhöhte Myopathiegefahr bei Kombination mit Statinen. }\end{array}$ \\
\hline Omega-3-Fettsäuren & $\begin{array}{l}\text { Einzelabwägung, evtl. nach Erreichung der LDL-Cholesterin-Zielwerte bei sehr hohem Risiko und } \\
\text { weiter bestehender Hypertriglyzeridämie; zurückhaltender Einsatz, da bisher keine überzeugenden } \\
\text { Endpunktstudien in Kombination mit Statinen. }\end{array}$ \\
\hline MCT-Fette & Zur diätetischen Begleittherapie bei sehr hohen Triglyzeridwerten \\
\hline
\end{tabular}

moderate Statindosis (z. B. Atorvastatin 20 mg/d oder Rosuvastatin $10 \mathrm{mg} / \mathrm{d}$ ).

Auch wenn sich auf den ersten Blick deutliche Unterschiede zwischen den ADA- und den ESC-Empfehlungen zeigen, führt die Umsetzung in beiden Fällen dazu, dass die überwiegende Mehrheit der Patienten mit Diabetes mellitus mit Statinen behandelt werden sollte.

Zum Erreichen der oben genannten ESC-Zielwerte steht eine Stufentherapie von Statinen, Ezetimib und PCSK9-Antikörper zur Verfügung ( $\triangleright$ Abb. 1) [10]. Nach Ausschluss oder ggf. Behandlung sekundärer Ursachen einer Hyperlipidämie werden als Therapie der Wahl Statine eingesetzt. Wenn dies trotz ausreichender Dosis nicht genügt, um den individuellen Zielwert zu erreichen, erfolgt in einem nächsten Schritt eine Kombination mit Ezetimib und in dritter Stufe, insbesondere bei klinischer Progression einer kardiovaskulären Erkrankung, eine Kombination mit PCSK9-Inhibitoren. Laut Beschluss des Gemeinsamen Bundesausschusses (G-BA) müssen PCSK9-Inhibitoren durch einen Internisten mit Schwerpunktbezeichnung für Kardiologie, Nephrologie, Endokrinologie, Angiologie oder eine spezielle Lipidambulanz indiziert werden und können dann durch den Hausarzt weiter verschrieben werden. Als Ultima-Ratio-Therapie kann die regelmäßige Lipoproteinapherese eingesetzt werden. Diese sollte allerdings erst dann zum Einsatz kommen, wenn alle medikamentösen Ansätze ausgereizt sind. Wird klinisch die Indikation zur LDL-Apherese gestellt, dann ist laut Beschluss des G-BA alternativ die Gabe eines PCSK9-Inhibitors indiziert und wirtschaftlich. Bei Patienten, die sich bereits primär zur Senkung des LDL-Cholesterins in der Lipidapherese befinden, sollte durch die Gabe eines PCSK9-Antikörpers die Apheresefrequenz reduziert und sogar die Beendigung dieses Therapiekonzepts angestrebt werden [11].

\section{Therapiestrategien bei erhöhten Triglyzeriden}

Bei Hypertriglyzeridämie sowie zur Senkung der Triglyzeride bei kombinierter Hyperlipidämie stehen Lebensstilmaßnahmen sowie die Blutzuckereinstellung im Vordergrund [1]. Der Einsatz von Fibraten und hochdosierten Fettsäuren zur weiteren Absenkung deutlich erhöhter Triglyzeridwerte muss individuell entschieden werden, da Endpunktstudien in Kombination mit Statinen keinen klaren kardiovaskulären Nutzen gezeigt haben ( $\triangleright$ Tab.5).

\section{Therapiestrategien bei Sondersituationen}

\section{Erhöhte Lipoprotein(a)-Werte}

Erhöhte Lp(a)-Werte (oder auch niedrige HDL-Cholesterin-Spiegel) können zurzeit nicht gezielt medikamentös beeinflusst werden, daher muss in diesen Fällen das übrige Risikoprofil optimiert und so z. B. eine optimale Einstellung des LDL-Cholesterins angestrebt werden. Es ist wichtig zu berücksichtigen, dass ca. $20 \%$ der Lp(a)Konzentration bei der LDL-Cholesterin-Bestimmung miterfasst wird, d. h., dafür ist der LDL-Cholesterin-Wert zu „korrigieren“. Bei deutlich erhöhten Lipoprotein(a)-Werten (> $60 \mathrm{mg} / \mathrm{dl}$ ) und Nachweis einer über ein Jahr progredienten Atheroskleroseerkrankung trotz optimaler Kontrolle aller anderen Risikofaktoren kann eine regelmäßige Lipoproteinapherese zur Absenkung erhöhter Lipoprotein(a)-Werte durchgeführt werden.

\section{Statinintoleranz}

Bei Patienten mit Diabetes mellitus und Statinintoleranz sollte ähnlich vorgegangen werden wie bei Patienten ohne Diabetes und mit Statinintoleranz. Es sollten mindestens 3 verschiedene Statine eingesetzt werden, bevor eine Statinintoleranz diagnostiziert wird (Ausnahme: Rhabdomyolyse auf ein Statin - dann sollte ein zweites 
Statin nur sehr zurückhaltend eingesetzt werden). Bei vielen Patienten ist es möglich, eine niedrige Statindosis in Kombination mit Ezetimib einzusetzen und so den LDL-Cholesterin-Wert signifikant zu reduzieren. PCSK9-Inhibitoren werden von Patienten mit Statinintoleranz in hohem Prozentsatz vertragen und können bei Patienten mit sehr hohem Risiko und deutlicher Distanz zum Zielwert eingesetzt werden. Versorgungsdaten aus Deutschland zeigen, dass PCSK9-Inhibitoren zu 70-80\% bei Patienten mit Statinintoleranz eingesetzt werden.

\section{Schwere Hypertriglyzeridämie}

Bei Triglyzeridwerten über $1000 \mathrm{mg} / \mathrm{dl}$ ist das Risiko für eine akute Pankreatitis deutlich erhöht [12]. Mit einer konsequenten Umsetzung von Lebensstilmaßnahmen (Alkoholkarenz, weitgehender Verzicht auf schnell verstoffwechselbare Kohlenhydrate) und einer strengen Blutzuckereinstellung gelingt es meist, die Werte deutlich abzusenken. Um das Pankreatitisrisiko bei schweren Hypertriglyzeridämien zu minimieren, können Fibrate und/ oder hochdosiert Omega-3-Fettsäuren eingesetzt werden, die die Triglyzeridwerte deutlich reduzieren. Kommt es bei Triglyzeridkonzentrationen über 1000 mg/dl zu einer akuten Pankreatitis, stellt die Plasmapherese eine Behandlungsoption dar, um die Triglyzeridkonzentration rasch abzusenken. Weitere Behandlungsoptionen stellen eine Gabe von Heparin und/oder Insulin (Aktivierung der Lipoproteinlipase) und Nahrungskarenz dar. Insbesondere bei sehr hohen Triglyzeridwerten kann ein diätetischer Ersatz von Nahrungsfetten durch MCT-Fette versucht werden.

\section{Fazit}

Kardiovaskuläre Ereignisse sind ein wesentlicher Grund für verfrühte Sterblichkeit und Multimorbidität der von Diabetes betroffenen Menschen. Die risikostratifizierte patientenbezogene LDL-Cholesterin-Senkung ist ein evidenzbasierter wichtiger integraler Bestandteil einer Diabetestherapie und kann die klinische Prognose unserer Patienten verbessern. Bei schwerer Hypertriglyzeridämie mit Werten über 1000 mg/dl senken Lebensstilmaßnahmen (Alkoholkarenz, weitgehender Verzicht auf schnell verstoffwechselbare Kohlenhydrate), Blutzuckereinstellung und eventuell zusätzlich Fibrate und/oder hochdosiert Omega-3-Fettsäuren die Triglyzeridkonzentration und damit das Pankreatitisrisiko deutlich.

\section{Gesellschaftsvertreter}

K.G. Parhofer und D. Müller-Wieland vertreten die DDG.

A.L. Birkenfeld vertritt die AG Diabetes und Herz.

W. Krone und M. Merkel vertreten die DGE.

M. Lehrke, N. Marx, K.S. Schütt und A. Zirlik vertreten die AG Herz und Diabetes der DGK.

N. Marx, W. Krone, D. Müller-Wieland vertreten die AG HerzHormone-Diabetes der DGK-DGE-DDG.
Interessenkonflikt

K.G.P. erhielt Vortragshonorare, Honorare für Advisory Board Tätigkeit, Honorare für DMC-Tätigkeit und/oder Forschungsunterstützung von folgenden Unternehmen: Aegerion, Akcea, Amarain, Amgen, BerlinChemie, Boehringer-Ingelheim, Ionis, MSD, Omniamed, Pfizer, Regeneron, Sanofi.

A.L.B. erhielt Vortragshonorare von Amgen, MSD und Sanofi. W.K. erhielt Vortragshonorare von Amgen, Bayer Vital, Boehringer Ingelheim, OmniaMed, Roche und Sanofi.

N.M. hat Unterstützung für die Leitung klinischer Studien von Boehringer Ingelheim erhalten, er fungiert als Berater von Amgen, AstraZeneca, Bayer, BMS, Boehringer Ingelheim, MSD, NovoNordisk und SanofiAventis und erhält Forschungsförderung von MSD und Boehringer Ingelheim. Zusätzlich hält er Vorträge für Amgen, AstraZeneca, Bayer, BMS, Boehringer Ingelheim, Lilly, MSD, NovoNordisk und Sanofi-Aventis. M.L. hat Forschungsgelder für experimentelle und klinische Studien von Boehringer Ingelheim und MSD erhalten; er fungierte als Berater für Boehringer Ingelheim, Sanofi-Aventis, MSD, AstraZeneca, Lilly, NovoNordisk, Amgen und Bayer sowie als Redner für Boehringer Ingelheim Sanofi-Aventis, MSD, AstraZeneca, Lilly, NovoNordisk and Bayer. M.M. hat Honorare und/oder Forschungsgelder von Amgen, BerlinChemie, MSD und Sanofi erhalten.

\section{Literatur}

[1] Catapano AL, Graham I, De Backer G et al. 2016 ESC/EAS Guidelines for the Management of Dyslipidaemias. Eur Heart J 2016; 37: 2999-3058

[2] Jacobson TA, Ito MK, Maki KC et al. National lipid association recommendations for patient-centered management of dyslipidemia: part 1full report. J Clin Lipidol 2015; 9: 129-169

[3] Jacobson TA, Maki KC, Orringer CE et al. National Lipid Association Recommendations for Patient-Centered Management of Dyslipidemia: Part 2. J Clin Lipidol 2015; 9 (6): S1 - S122 e1

[4] Jellinger PS, Handelsman Y, Rosenblit PD et al. American Association of Clinical Endocrinologists and American College of Endocrinology Guidelines for Management of Dyslipidemia and Prevention of Cardiovascular Disease - Executive Summary. Complete Appendix to Guidelines available at http://journals.aace.com. Endocr Pract 2017; 23: 479-497

[5] American Diabetes Association. 9. Cardiovascular Disease and Risk Management: Standards of Medical Care in Diabetes-2018. Diabetes Care 2018; 41 (Suppl. 1): S86-S104

[6] Gregg EW, Li Y, Wang J et al. Changes in diabetes-related complications in the United States, 1990-2010. N Engl J Med 2014; 370: 1514-1523

[7] Yusuf S, Hawken S, Ounpuu S et al. Effect of potentially modifiable risk factors associated with myocardial infarction in 52 countries (the Interheart study): case-control study. Lancet 2004; 364: 937-952

[8] Nordestgaard BG, Langsted A, Mora S et al. Fasting is not routinely required for determination of a lipid profile: clinical and laboratory implications including flagging at desirable concentration cut-points-a joint consensus statement from the European Atherosclerosis Society and European Federation of Clinical Chemistry and Laboratory Medicine. Eur Heart J 2016; 37: 1944-1958

[9] Sniderman AD, De Graaf ], Couture P et al. Regulation of plasma LDL: the apoB paradigm. Clin Sci (Lond) 2010; 118: 333 - 339

[10] Parhofer KG. The Treatment of Disorders of Lipid Metabolism. Dtsch Arztebl Int 2016; 113: 261-268

[11] Moriarty PM, Parhofer KG, Babirak SP et al. Alirocumab in patients with heterozygous familial hypercholesterolaemia undergoing lipoprotein apheresis: the ODYSSEY ESCAPE trial. Eur Heart J 2016; 37: 3588 - 3595

[12] Ewald N, Kloer HU. Treatment options for severe hypertriglyceridemia (SHTG): the role of apheresis. Clin Res Cardiol Suppl 2012; 7 (Suppl. 1): $31-35$ 